\title{
Datos enlazados para el análisis de la literatura grecolatina
}

\author{
Jorge Juan Linares-Sánchez*; Sonia Sánchez-Cuadrado**, Jorge Morato*** \\ *Universidad de Murcia \\ e-mail: jls12311@um.es | ORCID iD: https://orcid.org/0000-0002-2148-7669 \\ ** Universidad Complutense de Madrid \\ e-mail: sscuadrado@ucm.es | ORCID iD: https://orcid.org/0000-0002-7722-1982 \\ *** Universidad Carlos III de Madrid \\ e-mail: jmorato@inf.uc3m.es | ORCID iD: https://orcid.org/0000-0002-7530-9753
}

Recibido: 08-10-20; 2a versión: 26-01-21; Aceptado: 02-03-21; Publicado: 31-01-2022

Cómo citar este artículo/Citation: Linares-Sánchez, J.J.; Sánchez-Cuadrado, S.; Morato, J. (2022). Datos enlazados para el análisis de la literatura grecolatina. Revista Española de Documentación Científica, 45 (1), e 316. https://doi.org/10.3989/ redc.2022.1.1842

Resumen: Se describe la elaboración de una ontología de dominio para la representación de la literatura grecolatina en forma de datos enlazados. Se analizan los principios de la Web Semántica y la difusión semántica de contenido aplicados a la literatura clásica grecolatina. Se ha adaptado la metodología Methontology para la construcción de ontologías y se ha implementado un recurso en lenguaje formalizado. El resultado de esta investigación ha sido la elaboración de un proyecto piloto de datos enlazados basado en los principios y tecnologías Linked Open Data (LOD) en el campo de la literatura comparada, desarrollando la ontología Litcomp para la mejora del estudio acerca de la influencia y la pervivencia de la literatura grecolatina.

Palabras clave: ontología; Web Semántica; datos enlazados; literatura comparada; pervivencia literaria; literatura grecolatina.

\section{Linked data for the Greek and Latin literatura analysis}

Abstract: The development of a domain ontology for the Greek and Latin literature representation in the form of linked data is described. The principles of the Semantic Web and the semantic diffusion of contents applied to classical Greek-Latin literature are analyzed. The essence of the Methontology methodology has been adapted for the construction of ontologies and a formalized linguistic resource has been implemented. The result of this research has been the development of a pilot project of linked data based on the principles and technologies of Linked Open Data (LOD) in the field of comparative literature, in which the Litcomp ontology has been developed to improve the study of the influence and preservation of Greek and Latin literature.

Key words: ontology; Semantic Web; linked data; comparative literature; literary influences; Greek and Latin literature.

Copyright: (c) 2022 CSIC. Este es un artículo de acceso abierto distribuido bajo los términos de la licencia de uso y distribución Creative Commons Reconocimiento 4.0 Internacional (CC BY 4.0). 


\section{INTRODUCCIÓN}

El interés por avanzar en la investigación de la Literatura y la Filología ha motivado a los investigadores para explorar las tendencias en la transferencia de conocimiento y aprovechar metodologías innovadoras. En su evolución, las humanidades incorporan herramientas de análisis y gestión de la información que favorecen la generación de nuevos conocimientos y su difusión en la sociedad. Esta confluencia entre el saber humanístico y las TICs ha conformado las humanidades digitales, experimentando un intenso desarrollo en todas sus disciplinas (Baraibar, 2014a y 2014b; López Poza y Pena Sueiro, 2014; Schreibman y otros, 2016).

En concreto, en el ámbito de la influencia literaria, el análisis consiste en afirmar que un autor o un texto ha servido de inspiración a otro, al menos en el nivel más básico. Pero también es de gran interés el análisis interno de los textos que profundiza en las relaciones que se establecen entre ellos. La recreación de un texto anterior no constituye una mera copia, sino que requiere un procedimiento de creación artística, mediante el cual se seleccionan y transforman elementos literarios del texto origen para adaptarlos a la naturaleza y características del nuevo texto. Por eso, para la literatura comparada, resulta interesante identificar los elementos comunes de obras relevantes que muestran situaciones similares, incluso con los mismos personajes. En este caso para la investigación literaria es preciso establecer un marco con el que especificar la amplia gama de transformaciones experimentadas entre los textos, como cambios de personajes, de cronotopo o de género literario.

La identificación de influencias establece una representación conceptual que permite organizar los agentes que interactúan en las obras literarias. Un referente sobre estudios literarios e historiográficos es el proyecto Almahisto, que hizo confluir la representación semántica de los objetivos historiográficos con los literarios. Se centró en textos referentes al periodo 1700 a 1936. La plataforma del proyecto recoge el análisis historiográfico de eventos clave, mostrando los cambios de la narración a lo largo del tiempo (Morato, 2016).

Desde hace algunos años, las relaciones entre manifestaciones literarias en el ámbito digital se representan mediante datos enlazados (Berners-Lee, 2006; Heath y Bizer, 2011). En esta línea, un ejemplo representativo es la Biblioteca Virtual de Polígrafos que recoge obras de autores, datos bibliográficos y textos digitales. También establece relaciones semánticas que permiten expresar escuelas de pensamiento e influencias entre autores. Para ello se utilizan varios recursos como
EDM, la DBpedia y otros esquemas de metadatos. Otro ejemplo de la aplicación de principios de la Web Semántica es la Biblioteca Virtual Miguel de Cervantes. Cada registro bibliográfico tiene asignado una URI, que sirve para establecer relaciones con otros registros y colecciones. Estas aplicaciones ejemplifican como se puede enriquecer los datos de diversas fuentes de información mediante los recursos de la web semántica, presentándose de forma usable e intuitiva (Morato y otros, 2008). Asimismo, la BNE aplica datos enlazados para la representación formal de su catálogo, que se puede interrogar a través de un sistema de búsqueda documental basado en una ontología (BNE, 2014). Sin duda la aplicación de ontologías a las obras literarias no está exenta de dificultades debido a la limitación en la representación estética, la riqueza de las palabras y la variedad de personajes (Howell, 2002). Pero si se asume que el propósito es describir de forma parcial el universo literario, la representación de elementos esenciales y la relación de influencias formalizadas puede sin duda favorecer estudios de envergadura.

Por todo ello, el objetivo de este trabajo está dirigido a elaborar una representación conceptual sobre literatura comparada que permita expresar la pervivencia literaria de la cultura grecolatina. Se acomete un prototipo de estructura ontológica para facilitar la publicación colaborativa de registros que permita tejer una red global de influencia entre autores y obras de la literatura mundial. El estudio se inicia con un análisis de la aplicación de las humanidades digitales a los estudios grecolatinos y en concreto dirigidos a compartir mediante datos enlazados el conocimiento, de modo que favorezca la interoperabilidad con otros recursos. Después se presenta la metodología aplicada y, como resultado, la construcción de una ontología que permite la representación conceptual de las relaciones literarias grecolatinas.

\section{APLICACIÓN DE LAS HUMANIDADES DI- GITALES A LOS ESTUDIOS GRECOLATINOS}

Los estudios clásicos han atraído la atención de las humanidades digitales desde sus inicios (Barker y Terras, 2016). En relación con los estudios grecolatinos en el ámbito español se encuentran iniciativas de referencia lexicográfica en línea como el Diccionario Griego-Español codificado en TEI (Glorieux y Thuillier, 2010; Rodriguez-Adrados y otros, 2012; Thuillier, 2014). A nivel internacional en 1971, surgió el Thesaurus Linguae Graecae (TLG) constituyendo un banco de datos sobre literatura griega (TLG, 2014). Años más tarde, el TLG, unido a otras iniciativas como Eaqua (Büchler y otros, 2010, Schubert, 2011), facilitó la comparación de 
textos de literatura clásica mediante coocurrencia de frases y estadísticas. Barker y Terras (2016) señalaron que el TLG tenía ciertas deficiencias con respecto a los actuales sistemas de búsqueda, junto con una fuerte dependencia del soporte impreso frente al digital, destacando la necesidad de adaptar este recurso con las tecnologías actuales.

En relación con la recuperación documental, un importante proyecto internacional es Open Greek and Latin (OGL) ${ }^{1}$ que ofrece en acceso abierto ediciones digitales de la literatura grecolatina, acompañadas de traducciones, aparatos críti$\cos$ y otras utilidades filológicas. Asimismo, OGL engloba otras iniciativas de interés, como Homer Multitext Project, que recoge las versiones de los poemas homéricos para reflejar su evolución textual (Dué y otros, 2008). Otro proyecto que forma parte de OGL es la biblioteca digital Perseus. Esta biblioteca proporciona acceso a ediciones online de colecciones literarias, especialmente textos latinos y griegos, que pueden exportarse a lenguaje XML. Perseus también ofrece herramientas de análisis morfológico, traducciones, notas y comentarios (Smith y otros, 2000). Los registros de su catálogo se basan en FRBR (Functional Requirements for Bibliographic Records) englobado en el modelo entidad-relación LRM (Library Reference Model) de la IFLA. Este recurso identifica las diferentes entidades junto con sus relaciones en los registros bibliográficos (Babeu, 2019), favoreciendo el intercambio de información. Desde hace algunos años, se desarrolla un importante esfuerzo para adecuar los registros bibliográficos a las tecnologías de los datos enlazados. Perseus también participa en la Digital Latin Library (DLL), que persigue la catalogación y publicación de ediciones críticas digitales de obras latinas y el material asociado a ellas. Este proyecto facilita el intercambio de ideas sobre los textos a través de un sistema abierto de colaboración. Además, proporciona la serialización de su catálogo de acuerdo con las practicas LOD, por lo que supone una iniciativa relevante para la filología clásica.

Las propuestas para la comparación de textos aplican enfoques basados en las tecnologías de la Web Semántica. El proyecto Pelagio utiliza datos enlazados para representar la ubicación geográfica de yacimientos (Isaksen y otros, 2014) y permitir la navegabilidad entre las diferentes entidades. Por otro lado, la plataforma Recogito se encarga de anotar entidades del nombre a través de datos enlazados (Simon y otros, 2017). Sin embargo, a pesar de los esfuerzos todo apunta a que sigue faltando compatibilidad, por lo que el proyecto LiLa ha propuesto unificar los datos de diferentes recursos mediante el paradigma de datos enlazados de carácter lingüístico (LLOD) para el tratamiento textual del latín (Cecchini y otros, 2019).

Para los sistemas de búsqueda especializados, las taxonomías y los tesauros han tenido un papel destacado respecto a la normalización terminológica. Sin embargo, presentan ciertas características que reducen su capacidad para realizar inferencias y análisis detallado, como: la limitación para establecer diferentes tipos de relaciones; una definición imprecisa de las propiedades de cada concepto; la falta de capacidad para expresar axiomas complejos; o la deficiencia para expresar un espacio de valores. Estas carencias tratan de paliarse con la aplicación de ontologías formales (Guarino, 1995). Las ontologías tienen un papel notorio en la mejora de la representación conceptual y la inferencia de información (Gruber, 1993; Guarino, 1995). De entre los distintos tipos, Blums y Weigand (2016) destacan el papel de las ontologías de dominio (Guarino, 1998) para describir la representación conceptual para una determinada área de conocimiento o área temática específica. Para la construcción de ontologías resulta de especial utilidad la reutilización de elementos ya descritos en otros vocabularios y ontologías (Gómez-Pérez y otros, 2004), así como el conocimiento experto sobre conceptos y restricciones del dominio concreto. Este es el caso de la ontología de dominio HuCit, que incorpora una base de conocimientos sobre obras clásicas y metadatos de autores antiguos (Romanello y Pasin, 2017).

Con respecto a un ámbito más general, se han explorado otros recursos lingüísticos aplicados al análisis léxico de los textos. Alderuccio y Bordoni (2002) emplearon la red léxica Wordnet para optimizar el estudio de aspectos semánticos de textos literarios. En el proyecto Sharing Ancient Wisdo$m s^{2}$ se aventuraron a utilizar una ontología para la interrelación entre conceptos. La ontología SAWS permitió expresar las relaciones de los textos de los manuscritos de la colección entre sus diferentes versiones y traducciones (Jordanous y otros, 2012). También para el proyecto PRIN, Bartalesi y Meghini (2017) crearon una ontología para representar el conocimiento que un autor refleja sobre las obras de otro, a partir de los comentarios que realiza de ellas. Con esta ontología, los autores generaron registros sobre las fuentes primarias de Dante Alighieri. Por otro lado, Hastings y Schulz (2019) han planteado posibles soluciones ontológicas para expresar la dicotomía ficción-realidad, que se produce en la representación de los constituyentes de un texto literario, entre los personajes y sus atributos. Generalmente los participantes de un relato son ficticios, pero también son verosímiles, por lo que sus propiedades se corresponden 
con las de la realidad. Asimismo, en el proyecto POSTDATA $^{3}$ sobre la estandarización de la poesía se utiliza una ontología. En concreto, este proyecto propone un modelo ontológico desarrollado en OWL (Web Ontology Language) para facilitar la unificación de la terminología poética (ritmo, acento, tipología, etc.) a través de diferentes tradiciones literarias, periodos, géneros poéticos y autores. Para publicar los metadatos utilizan las tecnologías de la Web Semántica basadas en LOD.

En consecuencia, se constata la existencia de iniciativas que abordan las relaciones entre textos, ya sean enfocados a los comentarios acerca de otros autores o a la relación textual entre escritos. De igual modo, permanecen abiertas las líneas de investigación sobre las reelaboraciones literarias a través de la adaptación de los elementos de un texto anterior (personajes, motivos, etc.) para la creación de un nuevo relato.

\section{DESARROLLO METODOLÓGICO Y MATE- RIALES}

Con el objetivo indicado previamente, se exploraron los procedimientos para la construcción de ontologías formales. A falta de una técnica específica para la creación de ontologías en el campo de la literatura se ha aplicado una adaptación de la metodología Methontology (Gómez-Pérez y otros, 2004; Guzmán Luna y otros, 2012), por tratarse de una propuesta metodológica consolidada y de carácter generalista. También se tuvieron en consideración las recomendaciones de la guía de Noy y McGuinnes (2005), así como los fundamentos de los principios LOD (Hyland y Villazón Terrazas, 2011; Fernández-López y otros, 2019). El proyecto se ha acometido en tres fases a las que se superpusieron evaluaciones iterativas: 1) Análisis de requisitos y reutilización de elementos; 2) Selección de términos y relaciones; y 3) Implementación y publicación de la ontología. De manera transversal, durante el desarrollo de cada fase se ha procedido a la evaluación de la ontología mediante criterios formales para detectar y subsanar deficiencias.

En lo referente a las herramientas empleadas, la ontología se construyó con la aplicación Protégé de la Universidad de Stanford (Noy y otros, 2003), por tratarse de un software gratuito, de código abierto, de uso extendido y con las funcionalidades requeridas. Entre sus múltiples funcionalidades cuenta con SPARQL Query para la realización de consultas. $S P A R Q L$ y el razonador HermiT permiten evaluar la consistencia de la ontología. Otras herramientas usadas en la evaluación de la ontología han sido: 1) RDF Validator para analizar la sintaxis de los documentos RDF e identificar posibles errores ${ }^{4}$, y 2 )
OOps! (OntOlogy Pitfall Scanner!) $)^{5}$ para la evaluación de ontologías (Poveda-Villalón y otros, 2012). Para la publicación y difusión se ha utilizado KompoZer con el que se compusieron los registros en HTML. Por último, se ha empleado Lodview 6 para buscar y agregar la información disponible sobre el recurso a través de URIs desreferenciadas y ofrecer una presentación HTML del recurso RDF.

\subsection{Requisitos, fuentes y reutilización de ele- mentos}

La investigación literaria precisa que se especifiquen las transformaciones entre los textos, para lo que se precisa elaborar un vocabulario que permita añadir elementos de complejidad gradual. Por lo tanto, un requisito esencial de la ontología es permitir la expresión de información sobre los procesos de influencia textual en la literatura con diversos grados de especialización. La solución adoptada debe además admitir la creación de registros de datos enlazados adaptados a la especialidad.

Como fuente y documentación de base se escogió el estudio de la pervivencia de la literatura grecolatina en la que se aborda la influencia literaria de la Odisea. En concreto, el episodio sobre el viaje del héroe al más allá (la Nékyia del canto XI), que ha servido de inspiración y se ha reelaborado en otros textos de la literatura occidental desde su creación hasta la época actual (Linares Sánchez, 2020). Para determinar el dominio y la finalidad de la ontología se compiló una lista de diez preguntas de competencia que servirán para delimitar las pruebas posteriores.

- ¿Qué episodios elaboran elementos literarios de la obra $A$ ?

- ¿En qué autores ha ejercido influencia literaria el autor $A$ ?

- ¿Qué obras ha escrito el autor A?

- ¿En qué obras se han reelaborado elementos literarios de la obra $A^{\prime}$ ?

- ¿En qué episodios se han reelaborado elementos literarios del episodio a?

- ¿Qué episodios forman parte de la obra A'?

- ¿Qué personajes forman parte del episodio a?

- ¿En qué personajes ha ejercido influencia literaria el personaje a'?

- ¿El personaje $a^{\prime}$ y el personaje $b^{\prime}$ tienen la misma identidad?

- ¿El personaje $a^{\prime} y$ el personaje $b^{\prime}$ tienen diferente identidad? 
Un primer análisis de los requisitos y de la fuente principal para la construcción de la ontología indicó la posibilidad de reutilizar elementos de recursos como DBpedia, DC, RDA, VIAF y BNE, unos por su carácter específico para la literatura y otros por facilitar una interoperabilidad más extendida. DBpedia abarca términos especializados de todos los ámbitos del conocimiento (Auer y otros, 2007). A su vez, sus términos se utilizan en iniciativas de bibliotecas virtuales como la Biblioteca Virtual de Polígrafos o la Biblioteca Virtual Miguel de Cervantes que hacen uso de tecnologías LOD. Además, se incluyó DC y VIAF, por su carácter universal. Por último, el empleo del RDA Reference Element Sets proporcionó metadatos en RDF inherentes a la catalogación bibliográfica.

\subsection{Selección de términos y relaciones para la ontología Litcomp}

La selección del vocabulario para la ontología Litcomp se aborda con el propósito de crear registros con diferentes niveles de profundidad que representen la pervivencia literaria. Se inicia con una selección de términos de nivel genérico, para permitir establecer las relaciones entre autores y obras. Se continua con las propiedades que reflejan los vínculos básicos entre obras y autores, así como equivalencias y obras derivadas. Una vez establecido el primer nivel, se afronta la relación de registros entre datos enlazados especializados, y para ello se incorporan clases y propiedades adicionales. Estos elementos son tanto reutilizados como de elaboración propia, y han sido identificados a partir de la experiencia de especialistas en el campo de estudio de la literatura comparada. Sucesivamente se incorporan subniveles y relaciones específicas, como reelaboraciones de un texto anterior, hasta abarcar todos los elementos de la ontología Litcomp.

\subsection{Implementación y publicación}

La implementación y publicación de la ontología Litcomp se realiza en consonancia con las recomendaciones de la Web Semántica y con la aplicación de diversas herramientas de datos enlazados. Se construye la ontología con Protègè, que facilita el modelado y permite la exportación a RDF. También con KompoZer se elabora una versión en HTML para su publicación web y facilitar la difusión dirigida a los usuarios. Una vez interrelacionados todos los elementos de la ontología se generan las URIs válidas para poder ser ejecutadas en Lodview para conectar con recursos de diferentes fuentes.

\subsection{Evaluación}

Durante el desarrollo de cada fase, se revisa el estado de la ontología a lo largo de todo su ciclo de vida. En lo referente a su contenido, se analizan las características de completitud, consistencia, concisión, extensibilidad y sensibilidad de acuerdo con la metodología adoptada (Gómez-Pérez y otros, 1995; Gómez-Pérez, 1996). Según Gómez-Pérez (2001) son frecuentes los errores con estos criterios. Para evitarlos o subsanarlos se ejecutan procedimientos de evaluación, como la comprobación de una lista de chequeo, el uso de razonadores o la ejecución de casos prueba a fin de determinar el cumplimiento de las preguntas de competencia (López Rodríguez y otros, 2018). Para analizar las deficiencias usuales en la construcción de ontologías se ha empleado la aplicación OOPS! (Poveda-Villalón y otros, 2012). Asimismo, la comprobación de la correspondencia entre el modelo RDF y su visualización se examina mediante el servicio RDF Validation Service. Por último, la consistencia técnica se ha validado mediante el razonador HermiT.

\section{RESULTADOS}

\subsection{Reutilización de etiquetas para la identifi- cación de vocabulario}

Como resultado del análisis de requisitos y de la fuente principal se han seleccionado una serie de etiquetas de recursos análogos empleados en otros proyectos de humanidades digitales. El criterio seguido para la reutilización de etiquetas de otros recursos se ha determinado con el fin de favorecer la interoperabilidad, y por ello se han escogido los más utilizados en el área según estudios previos (Morato, 2016; Palacios y otros, 2006). Finalmente, la ontología Litcomp incorpora 17 etiquetas de los siguientes recursos:

- Ontología DBpedia. Se han seleccionado elementos relacionados con el campo de la literatura y reutilizados en otros proyectos: como persona (dbo:Person), escritor (dbo:Writer), obra (dbo:Work) o personaje de ficción (dbo:FictionalCharacter). Para expresar la influencia literaria entre autores se tomaron otras relaciones definidas en la ontología: como dbo:influenced y dbo:influencedBy. De esta manera es posible reflejar la evolución del pensamiento y las relaciones entre escritores pertenecientes a diversas épocas y tradiciones literarias.

- Dublin Core. Del modelo de metadatos DC se han utilizado los elementos que permiten aportar datos de entidades literarias, tales como su descripción (dcterms:description), temática (dcterms:subject), división (dcterms:hasPart, dcterms:isPartOf) o referencia bibliográfica (dcterms:bibliographicCitation). 
- RDA Reference Element Sets. Se ha habilitado el Unconstrained element sets, sin especificación de dominio ni rango, lo que ha favorecido una aplicación menos restringida. Los elementos del RDA Registry relacionados con la literatura comparada han sido: persona (rdac:Person), obra (rdac:Work), autoría (rdau:authorOf y su inverso rdau:author), y derivación (rdau:derivative y su inverso rdau:basedOn).

- VIAF y BNE. Se ha adaptado el uso de los ficheros de autoridades como datos enlazados de VIAF, y de los registros del catálogo bibliográfico y de autoridades de datos.bne.es para la localización, identificación y enlace a URIs de autores y obras.

\subsection{Organización de conceptos y relaciones de la ontología Litcomp}

Se ha diseñado una ontología que permite representar diferentes conceptos mediante distintos niveles jerárquicos y relaciones. En un primer nivel genérico, se han incluido las relaciones de influencia literaria entre autores y obras. De esta manera, se indica en forma de datos enlazados que un escritor ( $d b o:$ Writer) es el autor (rdau:authorOf) de una obra ( $d b o:$ Work). También que el autor o la obra, por ejemplo, Homero y su Odisea, han ejercido influencia literaria en otro autor (dbo:influenced) u obra (rdau:derivative), como Virgilio y la Eneida. dbo:Writer es una subclase de $d b o: P e r s o n$ lo que permite la inferencia. Se ha establecido la equivalencia (owl:equivalentClass) entre $d b o:$ Work y rdac:Work, y entre $d b o: P e r s o n$ y rdac:Person. Además, se ha habilitado la posibilidad de ofrecer otros datos como: el nombre del

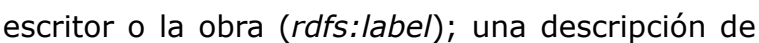
estos (dc:description); la etapa literaria a la que pertenecen (dc:subject); y un enlace a otras fuentes de información relevantes (rdfs:seeAlso). Los elementos empleados en este primer nivel han sido tomados de ontologías consolidadas, siguiendo las recomendaciones LOD (Hyland y Villazón Terrazas, 2011; Fernández-López y otros, 2019).

Las relaciones entre registros de datos enlazados especializados son resultado de la elaboración propia como: litcomp:Text, litcomp:InfluencedSame, litcomp:Episode y litcomp:InfluencedAnother. La Figura 1 muestra las relaciones entre dos obras. Además de las clases "escritor" y "obra", se han añadido "personaje de ficción" (dbo:FictionalCharacter) y "episodio" (litcomp:Episode), a las que se asociaron propiedades que permiten su localización. Tanto litcomp:Episode como dbo:Work y rdac:Work son subclases de litcomp:Text. Mediante dcterms:hasPart se han indicado los episodios y personajes de los que está compuesto un texto. Con dcterms:bi-
bliographicCitacion se ofrece la referencia bibliográfica completa. Esto ha permitido codificar relaciones de transtextualidad entre partes de una obra, como la que se produce entre la evocación de los muertos en Odisea XI y la catábasis de Eneas en Eneida VI. Además de obras, episodios y autores, es posible establecer la influencia entre los personajes de varios textos. El personaje de un texto puede reaparecer en un nuevo texto (litcomp:InfluencedSame), pero también se permite representar la influencia de un personaje con una identidad distinta, que reelabora el rol del anterior (litcomp:InfluencedAnother). Este sería el caso de Eneas, héroe inspirado en el Ulises de Homero.

Por otro lado, se han creado propiedades para especificar una clase concreta de derivación que consiste en que los elementos literarios de un texto (una obra o un episodio) son reelaborados en un texto posterior. Litcomp:HasReworking ha sido definida como una subpropiedad de rdau:derivative, con la posibilidad de detallar su tipología especializada según diversos enfoques.

1) Personajes y cronotopo. Un texto puede tener una reelaboración homodiegética (litcomp:HasHomodiegeticReworking), si el nuevo texto mantiene la ambientación y los personajes del texto original; heterodiegética (litcomp:HasHeterodiegeticReworking), si cambian; o mixta (litcomp:HasMixReworking), si se combinan.

2) Tipología textual. Cuando los elementos literarios de un texto se reelaboran en un nuevo texto se adaptan a otra tipología textual según este último pertenezca al género narrativo (litcomp:HasNarrativeReworking), teatral (litcomp:HasTheatricalReworking) o lírico (litcomp:HasPoeticReworking). A su vez, la adaptación narrativa tiene como subclases litcomp:HasEpicReworking para la epopeya y litcomp:HasNovelisticReworking para la novela.

Los nuevos términos, siguiendo las recomendaciones de los datos enlazados, han sido jerarquizados y asociados a vocabularios ya consolidados, evitando que queden aislados. En las Figuras 2 y 3 se recogen las jerarquías de las clases y propiedades de la ontología.

Las propiedades de la ontología cuentan con las propiedades inversas asociadas ( $d b o:$ influenced $B y$, rdau:basedOn, litcomp:InfluencedBySame, litcomp:InfluencedByAnother, litcomp:IsReworking, litcomp:IsNarrativeReworking, etc). De esta manera es posible indicar, por ejemplo, tanto que un texto tiene una reelaboración homodiegética posterior (litcomp:HasHomodiegeticReworking), como que un texto es una reelaboración homodiegética de otro anterior (litcomp:IsHomodiegeticReworking). 
Figura 1. Descripción de las relaciones entre Odisea XI y Eneida VI en la ontología Litcomp

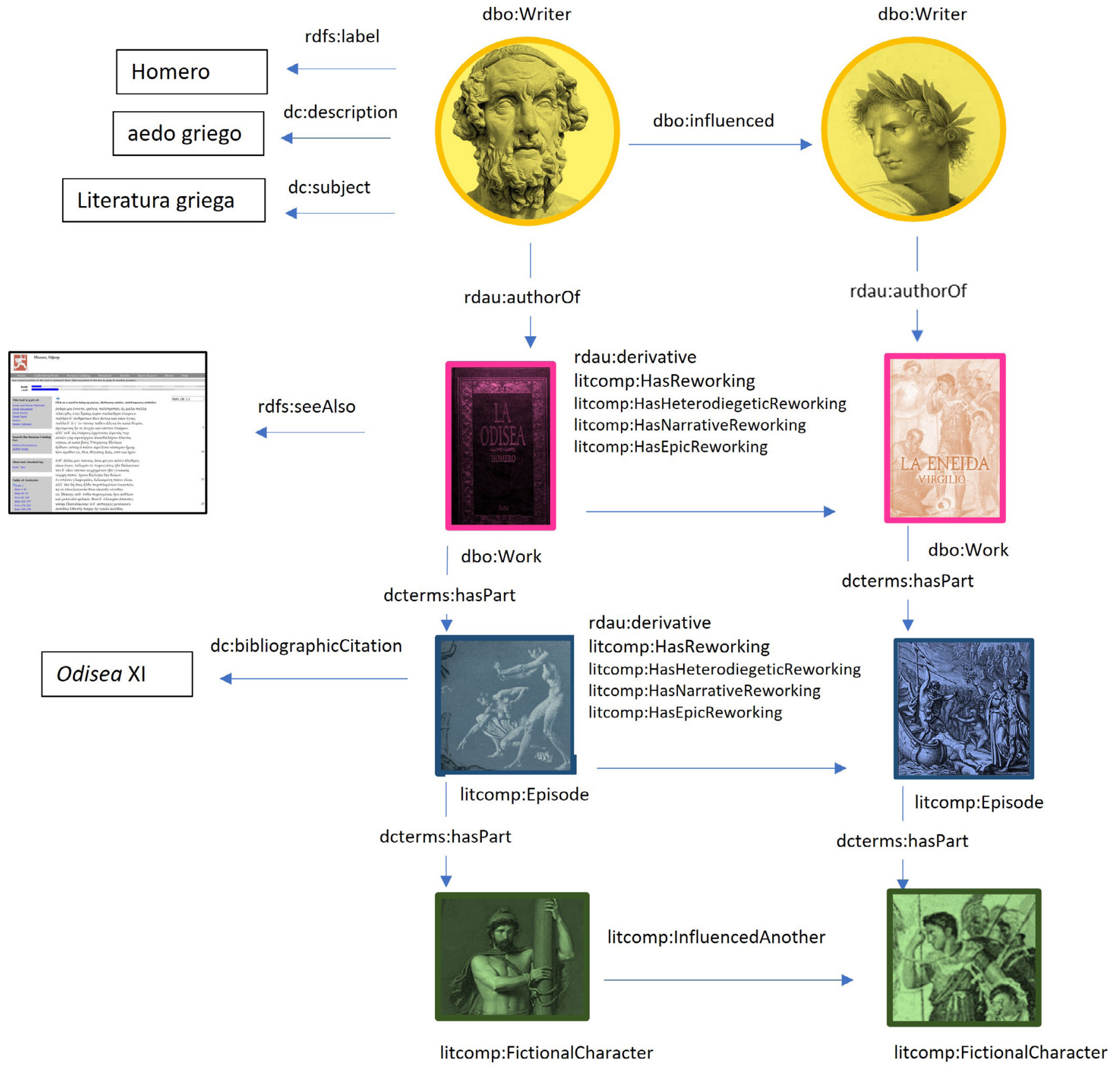

Figura 2. Jerarquía de las clases

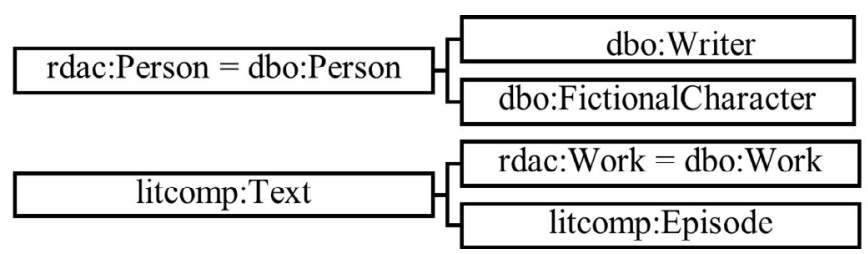


Figura 3. Jerarquía de las propiedades
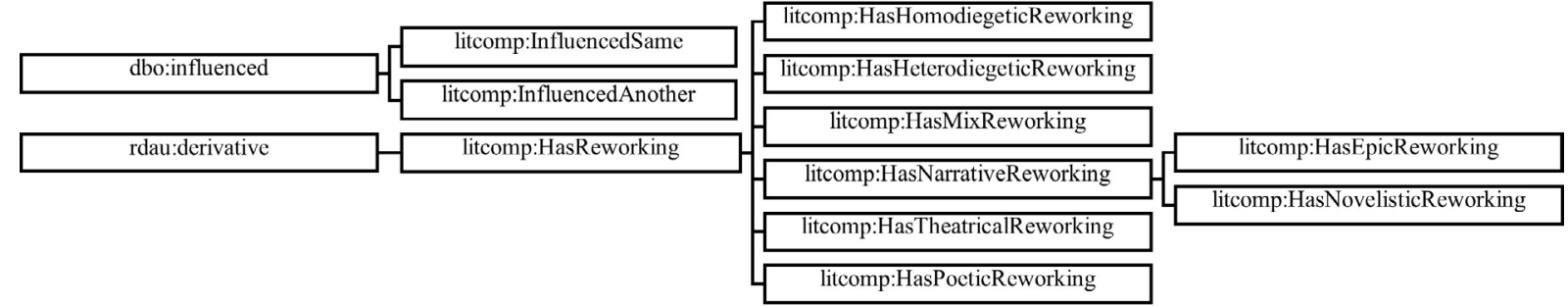

El vocabulario permite realizar aseveraciones específicas y académicas acerca de la pervivencia literaria. Por ejemplo, la Odisea de Javier Negrete (Negrete, 2019) es una obra que reelabora la Odisea de Homero, mantiene la ambientación y los personajes de la epopeya (litcomp:IsHomodiegeticReworking), pero los adapta a una nueva tipología textual, la novela (litcomp:IsNovelisticReworking). Adicionalmente, es posible identificar personajes de una nueva obra, como la maga Circe, que han sido tomados de una obra anterior (litcomp:InfluencedBySame); aunque no se conserve la ambientación ni los personajes, y se adapten los roles y las acciones literarias, como es el caso del Ulysses de Joyce (Joyce, 2008), se puede especificar esta relación e indicar que el papel de un personaje está basado en otro anterior (litcomp:InfluencedByAnother). Asimismo, es posible destacar que determinados episodios paralelos derivan (rdau:basedOn) de un mismo relato folclórico, como el del cíclope Polifemo en la Odisea (Homer, 2018) y el del gigante en Simbad el Marino (Dulac, 2014). La ontología permite establecer estas relaciones entre obras (rdac:Work), episodios (litcomp:Episode) o cualquier fragmento de texto (litcomp:Text).

El resultado de este proceso de diseño ha sido la base de una ontología Litcomp compuesta por 8 clases y 35 propiedades. Se han reutilizado 6 clases y se han creado 2 clases nuevas. Con respecto a las propiedades, 22 son de nueva creación. En total, la ontología consta de 181 tripletas RDF (Tabla I).

Tabla I. Clases y propiedades de la ontología Litcomp

\begin{tabular}{|l|c|}
\hline \multicolumn{1}{|c|}{ Tipo de dato } & Número \\
\hline Clases & 8 \\
\hline - Clases nuevas & 2 \\
\hline - Clases reutilizadas & 6 \\
\hline Propiedades de clase & 35 \\
\hline - Propiedades nuevas & 22 \\
\hline - Propiedades reutilizadas & 13 \\
\hline Total tripletas & 181 \\
\hline
\end{tabular}

\subsection{Producción y Publicación de los registros}

A partir de esta ontología base, y tomando como fuente el citado documento de investigación, se han creado 135 registros, organizados en cuatro clases según el recurso descrito fuera una persona, una obra, un episodio o un personaje. El conjunto de datos consta de 334 entidades. Se han establecido 247 relaciones de equivalencia entre entidades y 270 propiedades de clase. También, se han incluido 946 aserciones de anotación. La suma de las tripletas de estos registros asciende a 1.464 (Tabla II).

Tabla II. Elementos resultantes de la ontología Litcomp

\begin{tabular}{|l|c|}
\hline \multicolumn{1}{|c|}{ Tipo de dato } & Número \\
\hline Registros & 135 \\
\hline Entidades & 334 \\
\hline Relaciones equivalencia & 247 \\
\hline Propiedades de clase & 270 \\
\hline Aserciones & 946 \\
\hline Total tripletas & 1.464 \\
\hline
\end{tabular}

La especificación completa de la ontología está disponible tanto en versión $\mathrm{HCI}^{7}$ como en $\mathrm{XML} /$ $\mathrm{RDF}^{8}$. El recurso está alojado en litcomp.es. La página principal presenta la descripción el proyecto. También está documentado el conjunto de los registros creados con la ontología a través de un documento codificado en VoID. Desde la página principal se posibilita la navegación entre los diferentes registros, según su categoría: autores, obras, episodios y personajes. Todos ellos están conectados entre sí mediante enlaces, de manera que tejen una extensa red de pervivencia literaria. Por ejemplo, el registro HTML de Homero, el autor en el que se focaliza la investigación transformada en datos enlazados, se visualiza en una página con información sobre el aedo griego y su pervivencia literaria ${ }^{9}$. Los datos aparecen organizados en los siguientes apartados: 
- Nombre: Homero.

- Literatura asociada: Literatura griega.

- Descripción del autor. Información extraída de la Wikipedia y de fuentes académicas.

- Autores influenciados por Homero: Virgilio, Silio Itálico, Luciano, Ronsard, Lope de Vega, Stephen Phillips, Joyce, Nikos Kazantzakis, Carlota O’Neill,
Massimo Manfredi, Rick Riordan, etc. Pertenecen a diversas épocas y literaturas.

- Recursos LOD sobre Homero: Enlaces a su registro en datos.bne, DBpedia, VIAF y Wikidata.

- Obras de Homero registradas: La Odisea.

El registro HTML está asociado a una versión en RDF. En el siguiente cuadro reproduce una muestra reducida del documento:

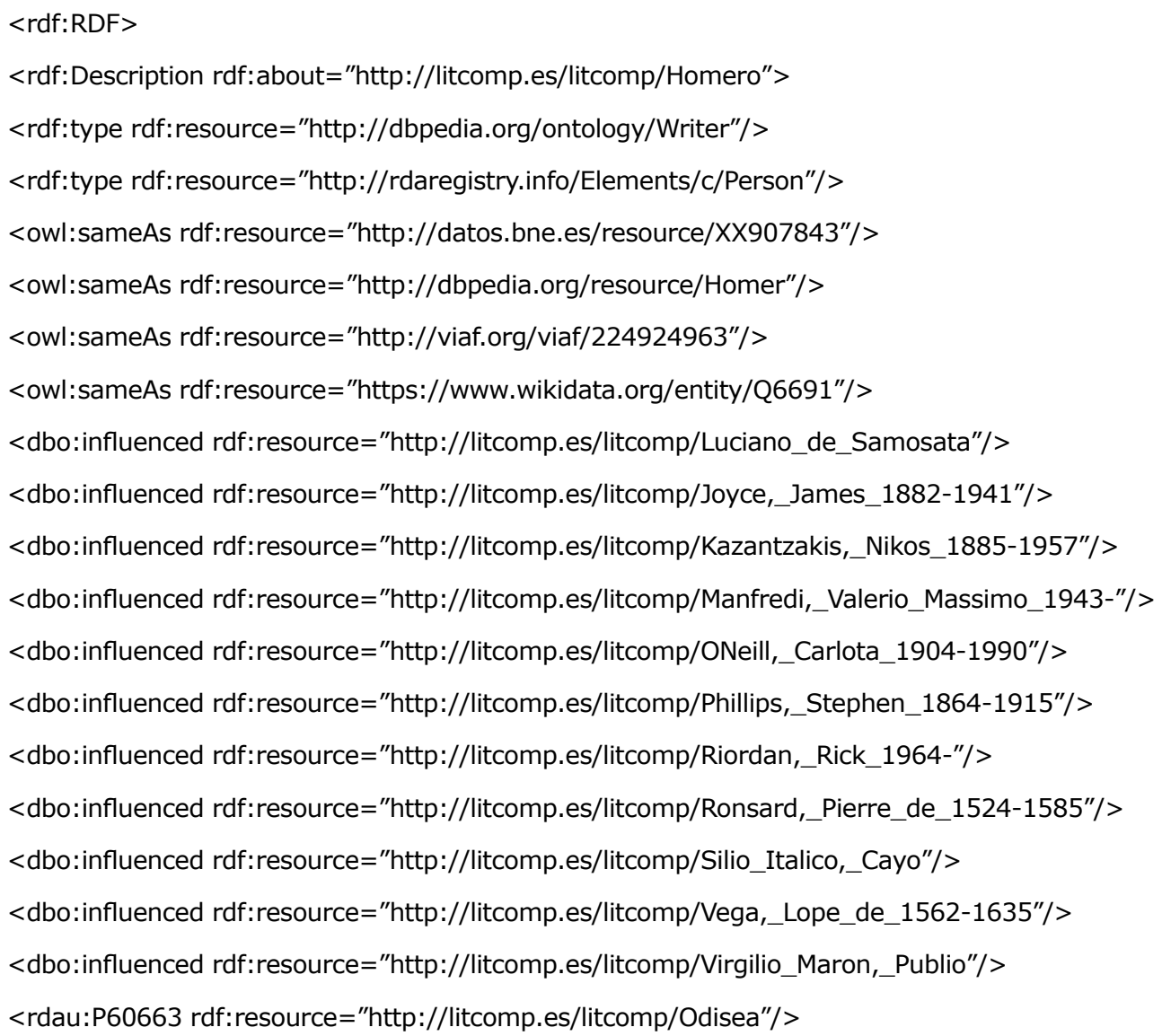
nombre dado al aedo griego antiguo a quien tradicionalmente se le atribuye la autoría de las principales poesías épicas griegas - la Ilíada y la Odisea-. Desde el período helenístico se ha cuestionado si el autor de ambas obras épicas fue la misma persona; sin embargo, antes no solo no existían estas dudas sino que la Ilíada y la Odisea eran considerados relatos históricos reales. Es el pilar sobre el que se apoya la épica grecolatina y, por ende, la literatura occidental. [Wikipedia] </dcterms:description>

<dcterms:subject rdf:resource="http://datos.bne.es/resource/XX527881"/>

$<$ rdfs:label xml:lang="es" $>$ Homero $</$ rdfs:label $>$

$</$ rdf:Description $>$

$</$ rdf:RDF $>$ 
- rdf:type. Esta propiedad indica la clase a la que pertenece el recurso. En este caso, indica que Homero es una persona (rdac:Person) y un escritor (dbo:Writter). Es necesario explicitar el conocimiento más esencial para que se puedan efectuar las deducciones contextuales que realiza una persona.

- owl:sameAs. Vincula la URI asignada a Homero con las de los otros recursos (datos.bne.es, VIAF, DBpedia y Wikidata). En el contexto de la Web Semántica, es esencial para facilitar la agregación de datos de diferentes fuentes.

- dbo:influenced. Establece una relación genérica de influencia, vinculada a las URI de autores posteriores.

- rdau: P60663 (authorOf). Proporciona la URI de aquellas obras del autor que han sido analizadas en cuanto a su pervivencia literaria. En el ejemplo, se establece que Homero es el autor de la Odisea.

- dcterms:description. Una breve descripción del autor en literales (literals, en RDF consisten en cadenas de caracteres), extraída de la Wikipedia.

- dcterms:subject. Especifica la literatura en la que se enmarca Homero u otros autores en forma de datos enlazados. Se han utilizado las URI del vocabulario controlado de datos.bne.es. En este caso, el identificador de la literatura griega es http://datos.bne.es/resource/XX527881.

- rdfs:label. Expresa en literales el nombre normalizado del autor a partir de los catálogos de control de autoridades (BNE y VIAF), salvo en ciertos casos en que existe otra versión del nombre que es más utilizada en el ámbito de estudio.

En el registro de obras, como la Odisea, aparecen elementos similares, también enlazados entre sí: nombre, etapa literaria, descripción, obras influenciadas, recursos LOD y episodios de los que hay registros. Se ha incorporado la posibilidad de acceder al texto original en el portal Perseus a través de sus URI codificadas según TEI. Los documentos relativos a los episodios ( $p$. ej. La Nékyia de la Odisea) presentan una configuración similar, con la especificación de los personajes que participan en el pasaje, como Agamenón. En la última categoría de registros, se indica además la influencia que el personaje ha ejercido o recibido.

La ejecución de la URI sobre Agamenon ${ }^{10}$ en Lodview genera automáticamente una página HTML con conexión a diferentes recursos. Su principal utilidad reside en la capacidad para identifi- car otros recursos albergados en la red que coinciden con el nuestro a través de las URI asociadas. En este caso, Agamenón, el personaje difunto que aparece en el canto XI de la Odisea, ha sido conectado con otros 105 recursos sobre el rey de Micenas. Esto resulta de especial relevancia en el ámbito de la investigación literaria, puesto que demuestra la capacidad de los datos enlazados de agregar información de diversas fuentes. El ejemplo de Lodview permite intuir el gran potencial que los datos enlazados podrían aportar a la investigación sobre literatura comparada.

\subsection{Evaluación}

La ontología ha sido examinada de forma reiterada a partir de una lista de comprobación de los errores más comunes. Para asegurar la corrección de la ontología en relación con la forma se ha implementado con el editor de ontologías Protégé. En las primeras revisiones se detectó y corrigió la existencia de clases aisladas, así como la falta de declaraciones de relación inversa y la poca exhaustividad en las propiedades. En lo relativo a las clases y propiedades de nueva creación, la aplicación detectó deficiencias debido a la ausencia de dominio y rango o de declaraciones de disyunción en algunos elementos. En ambos casos, se trata de decisiones de diseño. En lo referente a los rangos y dominios, solo se han definido los de aquellas propiedades que por su propia semántica lo exigen. Por ejemplo, litcom$p$ :InfluencedBySame relaciona un personaje con otro en cuya configuración ha influido, por lo que lógicamente ha de tener como dominio y rango la clase dbo:FictionalCharacter. Sin embargo, no ha sido definido para otras propiedades susceptibles de una aplicación más libre. Esto ocurre con propiedades como litcomp:IsNarrativeReworking, a la que se ha asignado litcomp:Text como dominio, pero no como rango.

Tras las diferentes modificaciones, otra de las comprobaciones ha validado la correspondencia del modelo RDF con su visualización mediante el servicio RDF Validation Service. La consistencia técnica se ha comprobado mediante el razonador HermiT. El resultado ha sido la ausencia de inconsistencias. Se ha verificado que la ontología permite responder a las preguntas de competencia predefinidas durante la fase de diseño, expresadas en lenguaje natural y derivadas de la experiencia académica en el campo. Con esta finalidad, se ejecutaron casos prácticos con los registros publicados. Mediante SPARQL Query de Protégé, las consultas se tradujeron a lenguaje SPARQL y se confirmó la corrección de la respuesta (ejemplo en Figura 4). 
Figura 4. Consulta en SPARQL: ¿Qué episodios elaboran elementos literarios de la Nékyia (Odisea XI)?

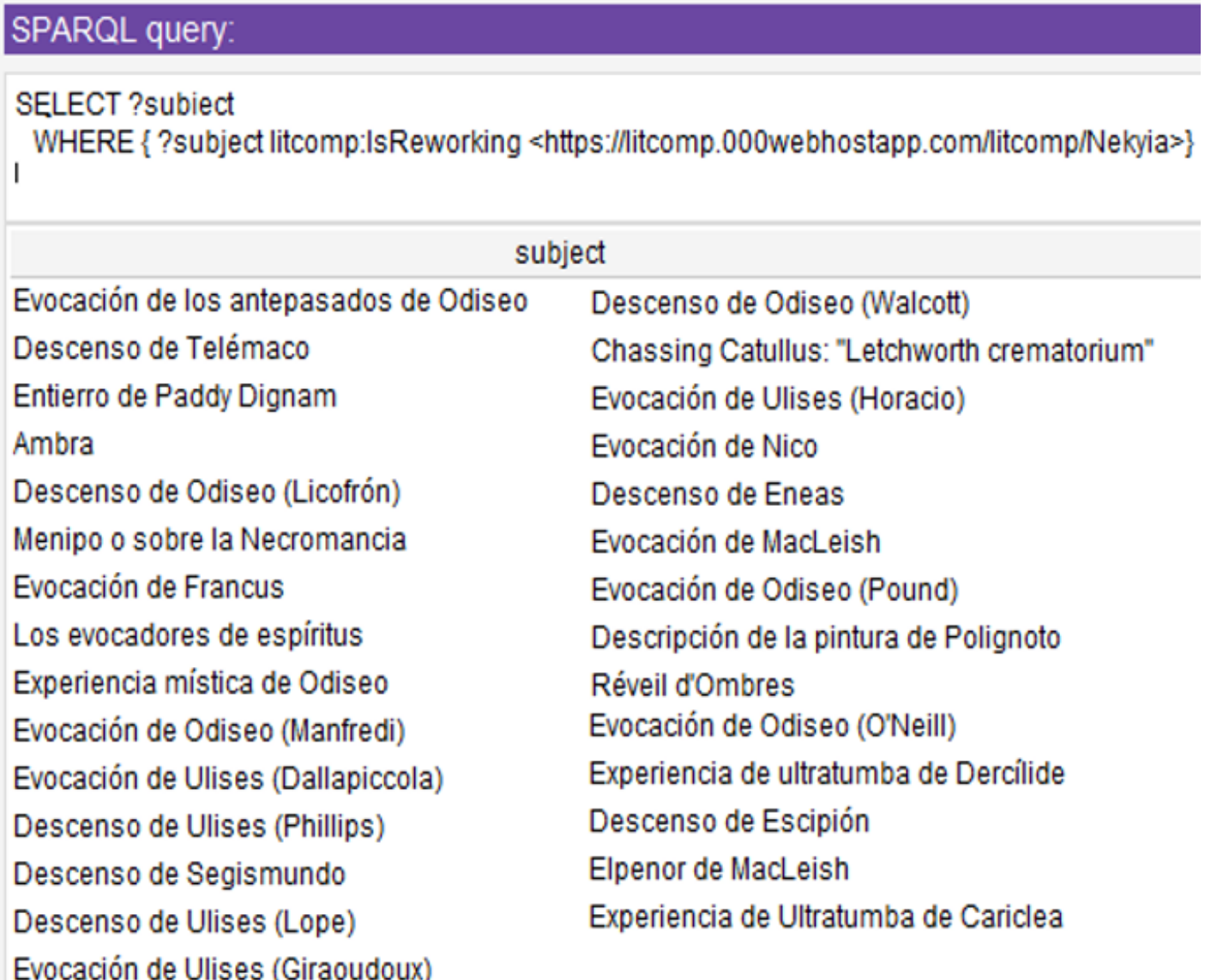

\section{CONCLUSIONES}

Algunas iniciativas genéricas han desarrollado funcionalidades que podrían ser de utilidad en el campo de la literatura comparada. Sin embargo, algunas de estas experiencias parecen haber sido abandonadas, incluso dejando de estar operativas. En Internet se encuentran múltiples aplicaciones LOD, aunque se evidencia una carencia significativa en lo que respecta a prototipos para este campo.

El resultado de este estudio aporta un nuevo recurso ontológico y establece un marco que propicia el análisis para investigaciones sobre literatura comparada. La ontología responde a las cuestiones habituales en este tipo de estudios a través de las preguntas de competencia predefinidas en la fase de diseño. El desarrollo de la ontología Litcomp aplica los principios y tecnologías de los datos enlazados al campo de la pervivencia literaria de la cultura grecolatina. Por todos estos motivos, esta propuesta resulta relevante en el ámbito de la investigación literaria. Destaca además la falta

de limitaciones del recurso, primero porque la ontología queda a disposición de la comunidad científica para su reutilización, y segundo porque el recurso se ha publicado para un perfil de usuario sin requisitos técnicos y por tanto accesible a un público amplio.

El problema analizado presenta múltiples facetas e interrelaciones. La falta de un vocabulario estandarizado ha favorecido una solución basada en el reuso e integración de etiquetas. El desarrollo de ontologías y vocabularios relacionados con la literatura, en especial los realizados en el ámbito de la biblioteconomía y la documentación, ha facilitado la creación de registros sobre la pervivencia literaria. Si bien ya existía una sólida base sobre la que trabajar, ha sido preciso crear nuevas clases y propiedades debido a la especialización de este campo. La ontología Litcomp reutiliza elementos de RDA y de DC según necesidades específicas, e incorpora términos de otros vocabularios para realizar referencias bibliográficas o adscripciones temáticas y temporales, permitiendo la fu- 
sión de los datos. Se usa vocabulario de otros recursos para reflejar la evolución de pensamiento y las relaciones. No obstante, la aplicación que se otorgado en la ontología Litcomp es más amplia, pues abarca a todos los autores de la literatura occidental y se puede extender a la literatura universal. El vocabulario permite realizar aseveraciones específicas sobre la pervivencia literaria y la ontología establece las relaciones entre obras, episodios y fragmentos de textos. El vocabulario semánticamente delimitado ha contribuido a la definición de consultas específicas. Aunque la muestra utilizada es reducida, se puede colegir su potencial como herramienta en el campo de la pervivencia literaria. Además de representar la reelaboración de un texto, esta propuesta es extensible a la descripción de diversas realidades como obras pictóricas, audiovisuales, musicales, cultura popular y experiencia vital. El recurso resulta escalable y potente, evitando una estructura compleja que limite su crecimiento y reutilización futura.

En conclusión, la ontología Litcomp se muestra en sintonía con los proyectos previos que ponen de manifiesto la necesidad de construir bancos de datos y estructuras de conocimiento sobre la literatura grecolatina, y que permitan aprovechar las ventajas de utilizar los principios de la Web Semántica y tecnologías LOD. Respecto a la diferencia con otras iniciativas, la ontología Litcomp proporciona la particularidad de estar adaptada expresamente para las obras de literatura y representar los tipos de influencias literarias mediante la identificación de elementos comunes. También permite representar la influencia literaria entre las obras a través de relaciones nuevas y la integración de recursos comunes en LOD.

\section{NOTAS}

1 Open Greek \& Latin https://www.opengreekandlatin. org/

2 Sharing Ancient Wisdoms. https://ancientwisdoms. ac.uk/

3 Poetry Standarization and Linked OpenData (POSTDATA) del Laboratorio de Innovación en Humanidades Digitales. http://postdata.linhd.uned.es/

4 Validation Service. https://www.w3.org/RDF/Validator/

5 OntOlogy Pitfall Scanner! http://oops.linkeddata.es/

6 LodView. https://lodview.it/

7 Ontología Litcomp. litcomp.es/ontology/litcomp.htm

8 Ontología Litcomp. litcomp.es/ontology/litcomp.rdf

9 Litcomp Homero. litcomp.es/litcomp/Homero.html

10 Litcomp Agamenón. http://litcomp.es/litcomp/Agamenon

\section{REFERENCIAS}

Alderuccio, D.; Bordoni, L. (2002). An Ontology-Based Approach in The Literary Research: Two Case-studies. En: González Rodríguez, M; y Suarez Araujo, C. P. (eds.), Proceedings of the Third International Conference on Language Resources and Evaluation (LREC'02), 1186-1190). Las Palmas, España: ELRA. Disponible en: http://sidenoter.nii.ac.jp/acl_anthology/browse/ L02-1030/2 [Fecha de consulta: 18/01/2021].

Auer, S.; Bizer, C.; Kobilarov, G.; Lehmann, J.; Cyganiak, R.; Ives, Z. (2007). DBpedia: A Nucleus for a Web of Open Data. En Aberer, K.; y otros (eds.), The Semantic Web, 722-735. Springer. https://doi. org/10.1007/978-3-540-76298-0_52

Babeu, A. (2019). The Perseus Catalog: of FRBR, Finding Aids, Linked Data, and Open Greek and Latin. En Berti, M (ed.), Digital Classical Philology, 53-72. https://doi. org/10.1515/9783110599572-005

Baraibar, A. (ed.). (2014a). Las Humanidades Digitales desde sus centros y periferias. En Humanidades Digitales: una aproximación transdisciplinar. Janus [en línea], Anexo 2. Disponible en: https://www.janusdigital.es/anexos/contribucion.htm?id=42 [Fecha de consulta: 22/07/2020].

Baraibar, A. (ed.). (2014b). Visibilidad y divulgación de la investigación desde las Humanidades Digitales. Experiencias y proyectos. Pamplona: Servicio de Publicaciones de la Universidad de Navarra. Disponible en: https://dadun.unav.edu/bitstream/10171/35705/4/ BIADIG_22_01_previas.pdf [Fecha de consulta: 22/07/2020].

Barker, E.; Terras, M. (2016). Greek Literature, the Digital Humanities, and the Shifting Technologies of Reading. En: Williams, G. (ed.), Oxford Handbooks Online: Scholarly Research Reviews, 1-25. Oxford: Oxford University Press. https://doi.org/10.1093/oxfordhb/9780199935390.013.45

Bartalesi, V.; Meghini, C. (2017). Using an Ontology for Representing the Knowledge on Literary Text: the Dante Alighieri Case Study. Semantic Web, 8 (3), 385-394. https://doi.org/10.3233/SW-150198

Berners-Lee, T. (2006). Linked Data [en línea]. Disponible en: http://www.w3.org/DesignIssues/LinkedData. html [Fecha de consulta: 22/07/2020].

BNE. (2004). BNE ontology Biblioteca Nacional de España [en línea]. Disponible en: http://datos.bne.es/def/ [Fecha de consulta: 22/07/2020].

Blums, I.; Weigand, H. (2016). Towards a Reference Ontology of Complex Economic Exchanges for Accounting Information Systems. 2016 IEEE 20th International Enterprise Distributed Object Computing Conference (EDOC): 1-10. https://doi.org/10.1109/ EDOC. 2016.7579388

Büchler, M.; Geßner, A.; Heyer, G.; Eckart, T. (2010). Detection of Citations and Textual Reuse on Ancient Greek Texts and its Applications in the Classical Studies: eAQUA Project. 5th Annual International Conference of the Alliance of Digital Humanities Organizations, DH 2010, London, UK, July 7-10, 2010, Conference Abstracts. Disponible en: http:// dh2010.cch.kcl.ac.uk/academic-programme/abstracts/papers/html/ab-637.html [Fecha de consulta: $10 / 02 / 2021]$ 
Cecchini, F. M.; Passarotti, M.; Marongiu, P.; Zeman, D. (2018). Challenges in Converting the Index Thomisticus Treebank into Universal Dependencies. Proceedings of the Second Workshop on Universal Dependencies (UDW 2018): 27-36. https://doi.org/10.5281/ ZENODO.2552203

Dué, C.; Ebbott, M; Scaife, A.R.; Sales, W.B.; Blackwell, C.; Smith Neel; Porter, D. C.; Baumann, R. (2008). The Homer Multitext Project. En: Digital Humanities 2008, 20th Joint International Conference of the Association for Literary and Linguistic Computing and the Association form Computers and the Humanities and the $1^{\text {st }}$ Joint International Conference of the Association for Literary and Linguistic Computing, the Association for Computer and Humanities, and the Society form Digital Humanities - Sociètè pour l'etude des medias interactifs, 5-11. Oulu, Finland: University of Oulu. Disponible en: http://www.ekl.oulu.fi/dh2008/Digital\%20 Humanities\%202008\%20Book\%20of\%20Abstracts. pdf [Fecha de consulta: 10/02/2021].

Dulac, E. (2014). Sindbad the Sailor \& Other Stories from The Arabian Nights (2014 [EBook \#47285]). The Project Gutenberg E-book. Disponible en: https://www. gutenberg.org/files/47285/47285-h/47285-h.htm\#Page_24 [Fecha de consulta: 10/02/2021].

Fernández-López, M.; Poveda-Villalón, M.; Suárez Figueroa, M. C.; Gómez-Pérez, A. (2019). Why are Ontologies not Reused across the Same Domain? Journal of Web Semantics, 57, 100492. http://dx.doi. org/10.2139/ssrn.3315457.

Glorieux, F.; Thuillier, S. (2010). Grec ancien, latin médiéval, balisage comparé de deux dictionnaires, vers des ressources linguistiques. Archivum Latinitatis Medii Aevi -Bulletin du Change (ALMA), 68, 161-181. http:// hdl.handle.net/2042/56776

Gómez-Pérez, A. (1996). Towards a Framework to Verify Knowledge Sharing Technology. Expert Systems with Applications, 11 (4), 519-529. https://doi. org/10.1016/S0957-4174(96)00067-X

Gómez-Pérez, A. (2001). Evaluation of Ontologies. International Journal of Intelligent Systems, 16 (3), 391409. https://doi.org/10.1002/1098-111X(200103)16: 3<391::AID-INT1014>3.0.CO;2-2

Gómez-Pérez, A.; Fernández-López, M; Corcho, O. (2004). Ontological Engineering: with examples from the areas of knowledge management, e-Commerce and the Semantic Web. London: Springer Verlag. https://doi.org/10.1007/b97353

Gómez-Pérez, A.; Juristo, N.; Pazos, J. (1995). Evaluation and assessment of knowledge sharing technology. En: Mars, N. J. I. (ed.), Towards very large knowledge bases: Knowledge building and knowledge sharing, 289296. Amsterdam: IOS Press.

Gruber, T. R. (1993). A translation approach to portable ontology specifications. Knowledge Acquisition, 5 (2), 199-220. https://doi.org/10.1006/knac.1993.1008.

Guarino, N. (1995). Formal ontology, conceptual analysis, and knowledge representation. International Journal of Human-Computer Studies 43 (5-6), 625-640. https:// doi.org/10.1006/ijhc. 1995.1066

Guarino, N. (1998). Formal Ontology and Information Systems. En: Guarino, N. (ed.), Proceedings of FOIS'98, 3-15. Amsterdam: IOS Press.
Guzmán Luna, J.; López Bonilla, M.; Torres, I. (2012). Metodologías y métodos para la construcción de ontologías. Scientia et Technica, 2 (50), 133-140. Disponible en: https://www.redalyc.org/pdf/849/84923878033. pdf [Fecha de consulta: 26/12/2021]

Hastings, J.; Schulz, S. (2019) Representing literary characters and their attributes in an ontology. En: Barton, A.; Seppala, S.; y Porello, D. (eds.), CEUR Workshop Proceedings, Joint Ontology Workshops JOWO 2019, 2518 Aachen: CEUR-WS. http://ceur-ws.org/ Vol-2518/paper-WODHSA4.pdf [Fecha de consulta: 22/07/2020]

Heath, T.; Bizer, C. (2011). Linked Data: Evolving the Web into a global data space. Synthesis Lectures on the Semantic Web: Theory and Technology, 1 (1), 1-136. https://doi.org/10.2200/S00334ED1V01Y201102WBE001

Homer. (2018). La Odisea (November 2, 2018 [EBook \# 58221]). The Project Gutember EBook. Disponible en: https://www.gutenberg.org/files/58221/58221-h/58221-h. htm [Fecha de consulta: 10/02/2021].

Howell, R. (2002) Ontology and the Nature of the Literary Work. Journal of Aesthetics and Art Criticism, 60 (1), 67-79. https://doi.org/10.1111/1540-6245.00053

Hyland, B.; Villazón Terrazas, B. (2011). Linked Data Cookbook [en línea]. Disponible en: https://www. w3.org/2011/gld/wiki/Linked_Data_Cookbook [Fecha de consulta: 22/07/2020].

Isaksen, L.; Simon, R.; Barker, E. T. E.; de Soto Cañamares, P. (2014). Pelagios and the Emerging Graph of Ancient World Data. En: Menczer, F.; Hendler, J.; y Dutton, W. (coords.), WebSci'14 Proceedings of the 2014 ACM Web Science Conference, 197-201. New York: ACM. https://doi.org/10.1145/2615569.2615693

Jordanous, A.; Lawrence, K; Hedges, M.; Tupman, C. (2012). Exploring Manuscripts: Sharing Ancient Wisdoms Across the Semantic Web. En: Burdescu, D. (coord.), Proceedings of the 2nd International Conference on Web Intelligence, Mining and Semantics, 1-12. New York: ACM. https://doi. org/10.1145/2254129.2254184

Joyce, J. (2008). Ulysses (August 1, 2008 [EBook \#4300]). The Project Gutenberg EBook. Disponible en: http://www.gutenberg.org/files/4300/4300-h/4300-h. htm [Fecha de consulta:10/02/2021]

Linares Sánchez, J. J. (2020). El tema del viaje al mundo de los muertos en la Odisea y su tradición en la literatura occidental. Murcia: Editum.

López Poza, S.; Pena Sueiro, N. (eds.). (2014). Humanidades Digitales: desafíos, logros y perspectivas de futuro. Janus, Anexo 1 [en línea]. Disponible en: https://www.janusdigital.es/anexo.htm?id=5 [Fecha de consulta: 22/07/2020].

López Rodríguez, Y. A.; Hidalgo-Delgado, Y.; Silega Martínez, N. (2018). Un método práctico para la evaluación de ontologías. En: Hidalgo Delgado, Y.; y Leiva Mederos, A. A. (eds.), CEUR Workshop Proceedings, 3rd International Workshop on Semantic Web IWSW 2018, 2096, 76-85. La Habana: CEUR-WS. http:// ceur-ws.org/Vol-2096/paper7.pdf [Fecha de consulta: 22/07/2020]

Morato, J. (2016). Aplicación de linked data y la web semántica a la investigación histórica. En: Romero, M.; y 
Colmenero, M.J., Historiografía digital, proyectos para almacenar y construir la Historia, Anejos de la Revista de Historiografía, 4, 117-138. Madrid: Instituto de Historiografía Julio Caro Baroja, Universidad Carlos III de Madrid.

Morato, J.; Sánchez-Cuadrado, S.; Fraga, A.; Moreno-Pelayo, V. (2008). Hacia una web semántica social. El Profesional de la Información, 17 (1), 78-85. https:// doi.org/10.3145/epi.2008.ene.09

Negrete, J. (2019). Odisea. Barcelona: Espasa Libros.

Noy, N. F.; Crubezy, M.; Fergerson, R. W.; Knublauch, H.; Tu, S. W.; Vendetti, J.; Musen, M. A. (2003). Protégé-2000: an Open-source Ontology-Development and Knowledge-Acquisition Environment. AMIA Annual Symposium Proceedings. 2003, 953. PMID: 14728458; PMCID: PMC1480139.

Noy, N. F.; McGuinness, D. L. (2005). Desarrollo de Ontologías-101: Guía para crear tu primera ontología [en línea]. Disponible en: https://protege.stanford.edu/ publications/ontology_development/ontology101.pdf [Fecha de consulta: 22/07/2020].

Palacios, V.; Morato, J.; Lloréns, J.; Moreiro, JA (2006). Indicadores web sobre utilización de ontologías. En: Cunha, M.: Rocha, A. (eds.), Actas da $1^{\text {a }}$ Conferência Ibérica de Sistemas e Tecnologias de Informação, CISTI 2006, vol II, 199-214. Lousada, Portugal: Associacao Iberica de Sistemas e Tecnologias de Informacao. Disponible en: http://www.cisti.eu/index. php?option $=$ com_content $\&$ view $=$ article\&id=60\&Itemi$\mathrm{d}=144$ \&lang=es [Fecha de consulta: 22/07/2020].

Poveda-Villalón, M.; Suárez-Figueroa, M.C.; Gómez-Pérez, A. (2012). Validating ontologies with OOPS!. En: ten Teije, A.; Völker, J.; Handschuh, S.; Stuckenschmidt, H.; d'Aquin, M.; Nikolov, A.; Aussenac-Gilles, N.; y Hernandez, N. (eds.), Knowledge Engineering and Knowledge Management. EKAW 2012. Lecture Notes in Computer Science, vol 7603, 267-281. Berlin: Springer Verlag. https://doi.org/10.1007/978-3-64233876-2_24.
Rodriguez-Adrados, F; Rodríguez Somolinos, J.; Gangutia, E.; Lara Nava, M. D.; Rodríguez Somolinos, H.; Berenguer Sánchez, J. A.; Arnaud-Thuillier, S.; Glorieux, F. (2012). Diccionario Griego-Español [en línea] [Data set]. Digital. CSIC. https://doi.org/10.20350/ DIGITALCSIC/4668

Romanello, M.; Pasin, M. (2017). Using Linked Open Data to Bootstrap a Knowledge Base of Classical Texts. Proceedings of the Second Workshop on Humanities in the Semantic Web (WHiSe II) co-located with 16th International Semantic Web Conference (ISWC 2017), 3-14. https://infoscience.epfl.ch/record/232930

Schreibman, S.; Siemens, R.; Unsworth, J. (eds.). (2016). A new companion to digital humanities. Oxford: Wiley. https://doi.org/10.1002/9781118680605

Schubert, C. (2011). Detailed description of eAQUA search portal. Working Papers Contested Order/ EAQUA Working Papers, 3, 33-53. https://doi.org/10.11588/ ea.2011.0.11567

Simon, R.; Barker, E.; Isaksen, L.; Cañamares, P. D. S. (2017). Linked Data Annotation Without the Pointy Brackets: Introducing Recogito 2. Journal of Map \& Geography Libraries, 13(1), 111-132. https://doi.org/ 10.1080/15420353.2017.1307303

Smith, D.; Rydberg-Cox, J.; Crane, G. (2000). The Perseus Project: a digital library for the humanities. Literary and Linguistic Computing, 15(1), 15-25. https:// doi.org/10.1093/Ilc/15.1.15

Thuillier, S. (2014). De una obra digitalizada a una obra digital: reflexiones en torno al Diccionario GriegoEspañol. En: López Poza, S.; y Pena Sueiro, N. (eds.), Humanidades Digitales: desafíos, logros y perspectivas de futuro. Janus [en línea], Anexo 1, 459-471. Disponible en: https://www.janusdigital.es/anexos/contribucion.htm?id=40 [Fecha de consulta: 22/07/2020].

TLG (2014). The History of the TLG®. [en línea] Disponible en: http://stephanus.tlg.uci.edu/history.php [Fecha de consulta: 05/02/2021] 